\section{Motivación, estrategias de aprendizaje y su relación con el nivel de habilidades investigativas en estudiantes de posgrado. Un estudio piloto}

\author{
Motivation, learning strategies and their relation with the level of \\ research skills in postgraduate students. A pilot study
}

\begin{abstract}
Resumen
Objetivo: Determinar la influencia de la motivación y el uso de estrategias de aprendizaje en el nivel de habilidades investigativas en estudiantes de posgrado de Odontología de la Universidad Nacional Mayor de San Marcos. Materiales y método: Se encuestaron 52 estudiantes que representan el 43,3\% del total, pertenecientes a los programas de maestría y especialidades. El 58,8\% de estudiantes eran del sexo masculino y el $41,2 \%$ del sexo femenino los cuales fueron seleccionados en forma aleatoria. Se utilizó el cuestionario de estrategias de aprendizaje y motivación CEAM y un cuestionario para evaluar las habilidades investigativas el cual fue previamente validado. Resultados: Se encontró asociación entre la motivación, estrategias de aprendizaje y habilidades investigativas de los estudiantes $\mathrm{OR}=0,14$ (IC 95\%: $0,04-0,6)$ y correlación moderada $r_{s}=0,64(p<0,001)$. Conclusiones: Los estudiantes de posgrado en Odontología tienen un nivel de habilidades investigativas, motivación y uso de estrategias de aprendizaje nivel medio, no se encontraron diferencias significativas en cuanto a las características sociodemográficas y educacionales de los estudiantes. Se concluye que los estudiantes que usan estrategias de aprendizaje y motivación tienen un 0,14 más probabilidades de tener mejores habilidades investigativas.

Palabras clave: Motivación; Estrategias; Aprendizaje; Educación de posgrado en Odontología; Estudiantes de Odontología.
\end{abstract}

\section{Abstract}

Objective: To determine the influence of motivation and the use of learning strategies in the level of investigative skills in postgraduate students of dentistry of the National University of San Marcos. Materials and method: Fifty-two students were surveyed representing $50 \%$ of the total, which belong to the programs of masters and specialties. $58.8 \%$ of the students were males and $41.2 \%$ of the females were randomly selected. The questionnaire of learning strategies and motivation CEAM and a questionnaire were used to evaluate the investigative skills which was previously validated. Results: We found an association between motivation, learning strategies and students' investigative abilities $\mathrm{OR}=0.14$ (IC 95\%: 0.04-0.6) and a moderate correlation between $r=0.64(p<0.001)$. Conclusions: Postgraduate students in dentistry have a level of investigative skills, motivation and use of mid-level learning strategies, no significant differences were found regarding the sociodemographic and educational characteristics of the students. It concludes that students using learning strategies and motivation are 0.14 more likely to have better investigative skills.

Keywords: Motivation; Strategies; Learning; Dental research; Dental education; Dental students.

\section{Artí́culo Original}

\author{
Dahiana Alfaro Carballido ${ }^{1, a, b}$, Teresa \\ Evaristo Chiyong ${ }^{2, a, c}$, Gerardo Ayala de \\ la Vega'a,a,d, César Palomino Castro ${ }^{3, e}$, \\ Roberto León-Manco ${ }^{4, a, f}$, Alberto \\ Quitzgaard Alvarez',
}

1 Facultad de Odontología. Universidad de San Martín de Porres. Lima, Perú.

2 Facultad de Odontología. Universidad Nacional Mayor de San Marcos. Lima, Perú.

3 Facultad de Educación. Universidad Nacional Mayor de San Marcos. Lima, Perú.

4 Facultad de Estomatología. Universidad Peruana Cayetano Heredia. Lima, Perú.

a Cirujano dentista.

b Maestra en Estomatología. Docente investigadora de posgrado.

c Magister en Odontoestomatología de Salud Pública.

d Doctor en Estomatología.

e Doctor en Educación. Docente investigador. Lic. en Filosofía.

f Maestro en Salud Pública.

g Doctor en Psicología. Psicopedagogo.

Correspondencia:

Dahiana Alfaro Carballido

Correo electrónico: lalfaroc@usmp.pe

Calle Crepúsculo 104 Dpto 301, Lima 1, Perú.

Coautores:

Teresa Evaristo Chiyong

tevaristoc@unmsm.edu.pe

Gerardo Ayala de la Vega gayala@unmsm.edu.pe

César Palomino Castro

palomino_unmsm@hotmail.com

Roberto León-Manco

roberto.leon@upch.pe

Alberto Quitzgaard Alvarez

lalbertoqwistgaard1@gmail.com

Conflicto de intereses: Los autores declaran no tener conflictos de interés.

Fuente de financiamiento: Autofinanciado.

Fecha de recepción: 24/04/17

Fecha de aceptación: 31/05/17

\section{Introducción}

El programa de posgrado de la Facultad de Odontología de la Universidad Nacional Mayor de San Marcos (UNM$\mathrm{SM}$ ) en la constante preocupación por la actualización y mejora de calidad en la educación universitaria posee una filosofía de formación integral, humanística, científica y social, sustentada en las teorías de aprendizaje constructivista cuyos ejes de integración son la investigación, atención integrada y la responsabilidad social, con una orientación académica crítica, reflexiva, métodos de estudio actualizados y participación activa del estudiante, que busca articular las disciplinas y desarrollar competencias profesionales en sus egresados poniendo énfasis en las habilidades investigativas en coherencia con el objetivo 
principal de la universidad que es ser generadora de nuevo conocimiento ${ }^{1-3}$.

La tendencia actual de la enseñanza es conocer las estructuras cognitivas de los estudiantes en el contexto del aula para abordar el proceso de enseńanza- aprendizaje en su totalidad y así poder orientar al estudiante hacia el aprendizaje significativo teniendo como protagonista principal al estudiante autónomo y capaz de desarrollar estrategias que le permitan "aprender a aprender" 4-6.

Hoy en día se están realizando varios estudios sobre cómo la motivación y uso de estrategias benefician el aprendizaje, siendo esta última una de las principales líneas de investigación en psicología educativa ${ }^{7-10}$. A la fecha no hay estudios que refieran la relación entre la motivación, estrategias de aprendizaje y nivel de habilidades investigativas.

Para evaluar la motivación y estrategias de aprendizaje se utilizó el Cuestionario de Estrategias de Aprendizaje y Motivación (CEAM) validado internacionalmente y aplicado en diferentes pesquisas en ciencias de la salud y en psicología educativa ${ }^{11,12}$. Las habilidades investigativas se evaluaron con un cuestionario de 45 ítems valorado en tres dimensiones de acuerdo a las etapas de investigación científica el cual fue previamente validado. Existen trabajos previos desarrollados en diferentes contextos $5,7,11,13$ y un estudio similar realizado en la facultad de educación de la UNMSM ${ }^{14}$, pero ninguno que se haya realizado en el posgrado en Odontología, por lo que se plantea determinar cómo la motivación y uso de estrategias de aprendizaje influyen en el nivel de habilidades investigativas de los estudiantes.

\section{Materiales y método}

Estudio de tipo descriptivo correlacional, se encuestaron 52 estudiantes que representaron el 43,3\% del total de estudiantes de posgrado, perteneciente a los programas de maestría y especialidades seleccionados en forma aleatoria. Previamente se solicitó la aprobación del proyecto por la unidad de posgrado y la autorización para la ejecución del trabajo, se cumplieron con las normas bioéticas que implican a la investigación científica.

Validez y confiablidad de los instrumentos y aplicación de los cuestionarios. Se utilizaron dos cuestionarios. El cuestionario CEAM que consta de 82 ítems y dos escalas una en referencia a la motivación que se subdivide en cinco dimensiones: motivación extrínseca, motivación intrínseca, valor de la tarea, confianza, eficacia personal y otra escala que evalúa las estrategias de aprendizaje que se subdivide en nueve dimensiones: el ensayo, la elaboración, la organización, pensamiento crítico, autorregulación metacognitiva, manejo de recursos, regulación al esfuerzo, aprendizaje en paralelo y búsqueda de ayuda. Las alternativas de respuesta son de tipo Likert en una escala de cinco puntos (1 nunca, 2 pocas veces, 3 algunas veces, 4 muchas veces, 5 siempre) el cual al procesarlo se recategorizó a alto, medio y bajo ${ }^{12}$.

Al sumar las puntuaciones de los 82 ítems se obtiene un máximo total de 410 puntos. De acuerdo con los resultados obtenidos al sumar las puntuaciones obtenidas por los individuos que respondieron el cuestionario, la puntuación final se interpreta de la siguiente manera: 1-136 bajo uso de estrategias de aprendizaje, 137-273 regular uso de estrategias de aprendizaje, 274-410 alto uso de estrategias de aprendizaje el cual se recategorizó a alto y bajo. Se aplicó la versión en español del cuestionario ya utilizada y validada a nuestro contexto por Villegas y cols ${ }^{12}$.

Para la elaboración de los ítems del cuestionario de habilidades investigativas se realizó una evaluación y análisis crítico de la literatura científica actualizada sobre el tema, el cuestionario está compuesto de 45 ítems subdividido en tres dimensiones según las etapas de investigación: planificación, ejecución y difusión de acuerdo al nivel alcanzado bajo, medio y alto con un puntaje total de 90 puntos interpretados de la siguiente manera: 0-30 bajo nivel de habilidades investigativas, 31-60 medio nivel de habilidades investigativas y del 61-90 alto nivel de habilidades investigativas. La validez de contenido se realizó mediante un juicio de 11 expertos con el grado de Doctor y Especialistas con experiencia en investigación científica y educación. Se obtuvo un valor de $\mathrm{V}$ de Aiken de 0,98 existiendo un buen nivel de acuerdo entre los jueces. El análisis de constructo se realizó aplicando el análisis factorial para lo cual se realizó la prueba de Kaiser-MeyerOlkin 0,534 y prueba de esfericidad de Bartlett dando $\mathrm{p}<0,001$ teniendo como resultado concordancia entre componentes ${ }^{14,15}$.

La confiabilidad de los Instrumentos se realizó mediante el Alfa de Cronbach por cada dimensión del cuestionario dando resultado un alfa de 0,967 para todo el cuestionario y 0,914, 0,948 y 0,909 para cada dimensión y un valor de Correlación de Spearman - Brown entre ítems de $1(\mathrm{p}<0,001)$ lo cual indicó una alta confiabilidad.

De la misma forma se evaluó la confiabilidad del CEAM obteniendo un valor de Alfa de Cronbach de 0,885 para todo el cuestionario.

Ambos cuestionarios fueron aplicados a los estudiantes del posgrado en Odontología de la UNMSM en el periodo de enero a junio del 2016 obteniendo el porcentaje de no respuestas de 13,3\% $(\mathrm{n}=8)$ y el tiempo utilizado promedio para desarrollar ambos cuestionarios fue de $25( \pm 2,35)$ minutos.

El procesamiento de la información se realizó con el programa estadístico SPSS 23, el análisis univariado de las variables se obtuvo mediante el análisis descriptivo y de frecuencias de los datos mediante porcentajes, promedios y desviación estándar, El análisis bivariado de variables cualitativas se realizó con la prueba de Chi cuadrado, Fisher y t de Student para variables cuantitativas previo análisis de normalidad de los datos y para analizar la relación entre los puntajes promedio de cada instrumento con los puntajes de sus subescalas se utilizó la correlación de Spearman Brown. Para establecer el grado de asociación entre la motivación y uso de estrategias de aprendizaje con el nivel de habilidades investigativas se realizó el análisis con Odds Ratio (OR).

\section{Resultados}

En cuanto a las características sociodemográficas de los estudiantes entrevistados, la mayoría fue del género masculino con un $58,82 \%(n=52)$ y $41,2 \%$ del género femenino; solteros $(69,2 \%)$. Del total de estudiantes, $54,3 \%$ pertenecieron al programa de maestría y el 45,6\% a especialidades, siendo procedentes de la UNMSM $(38,4 \%)$.

La mayoría de estudiantes refirió trabajos de investigación previos siendo los más frecuentes los posters y mesas clínicas con un $30,7 \%$, más del $70 \%$ de los estudiantes refirió no pertenecer a una asociación científica, $62 \%$ dice no haber recibido cursos de metodología y métodos de estudio previos y el 75\% de estudiantes maneja el idioma inglés como segunda lengua. El interés por realizar investigaciones es bastante alto $(86,6 \%)$ (Tabla 1).

El nivel de habilidades investigativas en estudiantes del posgrado en odontología fue medio con un valor de 55,8\%. Según etapas se encontró un mejor nivel de habilidades investigativas en la 
etapa de planificación $(55,6 \%)$ seguida de la etapa de difusión $(48,1 \%)$ y ejecución con un 4,4\% (Figura 1).

Los ítems más valorados fueron el 4, 34 y el 68 correspondiente al desarrollo del planteamiento del problema, redacción de conclusiones y uso de tecnologías para presentaciones de impacto (Tabla 2).

El cuestionario completo valoró las estrategias de aprendizaje y motivación en general como alto con un $(53,8 \%)$ la motivación final fue considerada como alta con $53,8 \%$ siendo la subescala valor de la tarea la más alta con un 53,8\% y la menos valorada la motivación intrínseca $(36,5 \%)$, las estrategias mejor valoradas fueron las de elaboración, pensamiento crítico y estrategia metacognitivas $(57,6 \%)$.y las menos valorada aprendizaje paralelo (42,3\%) (Tabla 3). Los ítems más valorados de la escala de motivación en las diferentes subescalas hacen referencia a buen nivel de motivación interna y externa, donde se le da una adecuada apreciación al valor de las tareas, nivel de autoeficacia y eficiencia personal, también se presenta la mejor valoración de las estrategias de aprendizaje a la presentación de esquemas y gráficos.

No se encontró relación entre el nivel de habilidades investigativas y las variables sociodemográficas e investigativas de los estudiantes de posgrado ( $p>0,05)$. Se encontró asociación entre el nivel

Tabla 1. Características educacionales en investigación de los estudiantes de posgrado en Odontología de la UNMSM

\begin{tabular}{|c|c|c|c|}
\hline & & $\mathrm{n}$ & $\%$ \\
\hline \multirow{10}{*}{$\begin{array}{l}\text { Trabajos científi- } \\
\text { cos realizados }\end{array}$} & Posters & 8 & 15,4 \\
\hline & Presentación oral & 2 & 3,9 \\
\hline & Mesa clínica & 11 & 21,2 \\
\hline & Artículos científicos & 1 & 1,9 \\
\hline & Patentes & 0 & 0 \\
\hline & Poster y artículos & 5 & 9,6 \\
\hline & Poster y mesa clínica & 16 & 30,8 \\
\hline & No & 4 & 7,7 \\
\hline & $\begin{array}{l}\text { Poster y presentación } \\
\text { oral }\end{array}$ & 1 & 1,9 \\
\hline & Todo menos patente & 4 & 7,7 \\
\hline \multirow{2}{*}{$\begin{array}{l}\text { Pertenencia a } \\
\text { asociaciones } \\
\text { cientificas }\end{array}$} & $\mathrm{Si}$ & 15 & 29,4 \\
\hline & No & 37 & 70,6 \\
\hline \multirow{3}{*}{$\begin{array}{l}\text { Cursos previos } \\
\text { de metodología } \\
\text { y métodos de } \\
\text { estudio }\end{array}$} & $\mathrm{Si}$ & 19 & 38 \\
\hline & No & 33 & 62 \\
\hline & Inglés & 43 & 75 \\
\hline \multirow[t]{2}{*}{ Idiomas } & Otros & 1 & 2,8 \\
\hline & Inglés y portugués & 8 & 22,2 \\
\hline \multirow{2}{*}{$\begin{array}{l}\text { Interés por } \\
\text { realizar investiga- } \\
\text { ciones }\end{array}$} & $\mathrm{Si}$ & 38 & 86,5 \\
\hline & No & 14 & 13,7 \\
\hline
\end{tabular}

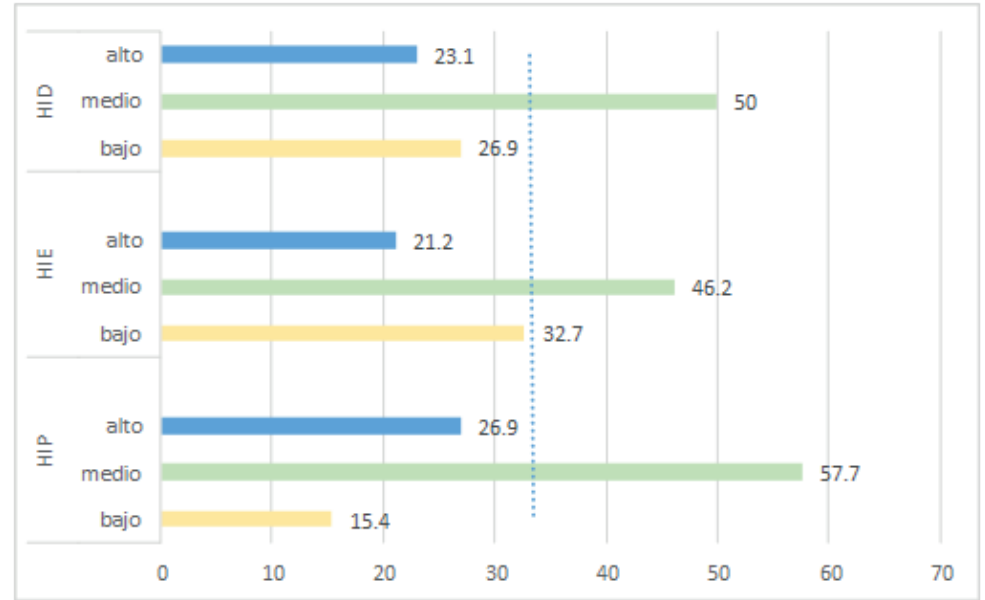

Figura 1. Nivel de habilidades investigativas en estudiantes de posgrado en la etapa de Planificación. HIP: Habilidades Investigativas de Planificación, HIE: Habilidades Investigativas de Ejecución, HID: Habilidades Investigativas de Difusión

de habilidades investigativas y la motivación de los estudiantes de posgrado empleando la prueba exacta de Fisher $(\mathrm{p}=0,004)$, así mismo con las subescalas motivación intrínseca $(\mathrm{p}=0,02)$, extrínseca $(\mathrm{p}=0,03)$, valor de la tarea

Tabla 2. Ítems más valorados por los estudiantes de posgrado en odontología de acuerdo a dimensiones de las habilidades investigativas

\begin{tabular}{|c|c|c|c|c|}
\hline Dimensiones & Items & $n$ & $\%$ & Interpretación \\
\hline & 1 & 37 & 71,1 & $\begin{array}{l}\text { Conoce sobre } \\
\text { epistemologia, ciencia } \\
\text { y método científico }\end{array}$ \\
\hline \multirow[t]{3}{*}{ Etapa de planificación } & 4 & 38 & 73,1 & $\begin{array}{l}\text { Desarrolla el plantea- } \\
\text { miento de investigación }\end{array}$ \\
\hline & 10 & 37 & 71,2 & $\begin{array}{l}\text { Utiliza organizadores, } \\
\text { esquemas , para sinte- } \\
\text { tizar la información }\end{array}$ \\
\hline & 15 & 37 & 71,2 & $\begin{array}{l}\text { Identifica los elementos } \\
\text { del diseño metodo- } \\
\text { lógico }\end{array}$ \\
\hline \multirow[t]{4}{*}{ Etapa de ejecución } & 34 & 33 & 63,5 & $\begin{array}{l}\text { Redacta ade- } \\
\text { cuadamente sus } \\
\text { conclusiones }\end{array}$ \\
\hline & 30 & 30 & 57,7 & $\begin{array}{l}\text { Selecciona y aplica } \\
\text { la prueba estadística } \\
\text { correcta }\end{array}$ \\
\hline & 31 & 30 & 57,7 & $\begin{array}{l}\text { Interpreta adecuada- } \\
\text { mente gráficos y tablas } \\
\text { estadisticas }\end{array}$ \\
\hline & 32 & 30 & 57,7 & $\begin{array}{l}\text { Determina con } \\
\text { precisión los resultados } \\
\text { de su estudio }\end{array}$ \\
\hline \multirow[t]{3}{*}{ Etapa de difusión } & 42 & 35 & 68,6 & $\begin{array}{l}\text { Usa adecuadamente la } \\
\text { tecnología para realizar } \\
\text { presentaciones de } \\
\text { impacto }\end{array}$ \\
\hline & 38 & 32 & 62,8 & $\begin{array}{l}\text { Conoce y realiza la } \\
\text { redacción de artículos } \\
\text { científicos }\end{array}$ \\
\hline & 43 & 31 & 60,8 & $\begin{array}{l}\text { Articula conocimientos } \\
\text { con facilidad para } \\
\text { elaborar un poster } \\
\text { científico. }\end{array}$ \\
\hline
\end{tabular}

$(\mathrm{p}=0,04)$, autoeficacia $(\mathrm{p}=0,004)$ y eficacia personal $(\mathrm{p}=0,015)$ (Tabla 4).

Se encontró asociación entre el nivel de habilidades investigativas y el uso de estrategias de aprendizaje y moti-

Tabla 3. Valoración del cuestionario de Motivación y Estrategias de Aprendizaje CEAM según dimensiones

\begin{tabular}{|c|c|c|c|}
\hline Sub escalas CEAM & & $\mathrm{n}$ & $\%$ \\
\hline \multirow{2}{*}{ Motivación intrínseca } & bajo & 33 & 63,4 \\
\hline & alto & 19 & 36,5 \\
\hline \multirow{2}{*}{ Orientación extrínseca } & bajo & 27 & 51,9 \\
\hline & alto & 25 & 48,0 \\
\hline \multirow{2}{*}{ El valor de la tarea } & bajo & 24 & 46,1 \\
\hline & alto & 28 & 53,8 \\
\hline \multirow{2}{*}{$\begin{array}{c}\text { Confianza en el control del } \\
\text { aprendizaje }\end{array}$} & bajo & 25 & 48,0 \\
\hline & alto & 27 & 51,9 \\
\hline \multirow{2}{*}{ Eficacia personal } & bajo & 26 & 50,0 \\
\hline & alto & 26 & 50,0 \\
\hline \multirow{2}{*}{ Motivación final } & bajo & 24 & 46,1 \\
\hline & alto & 28 & 53,8 \\
\hline \multirow{2}{*}{ El ensayo } & bajo & 25 & 48,0 \\
\hline & alto & 27 & 51,9 \\
\hline \multirow{2}{*}{ La elaboración } & bajo & 22 & 42,3 \\
\hline & alto & 30 & 57,6 \\
\hline \multirow{2}{*}{ La organización } & bajo & 23 & 44,2 \\
\hline & alto & 29 & 55,7 \\
\hline \multirow{2}{*}{ Pensamiento crítico } & bajo & 22 & 42,3 \\
\hline & alto & 30 & 57,6 \\
\hline \multirow{2}{*}{$\begin{array}{l}\text { Autorregulación meta- } \\
\text { cognitiva }\end{array}$} & bajo & 22 & 42,3 \\
\hline & alto & 30 & 57,6 \\
\hline \multirow{2}{*}{$\begin{array}{l}\text { Estrategias metacognitivas } \\
\text { total }\end{array}$} & bajo & 22 & 42,3 \\
\hline & alto & 30 & 57,6 \\
\hline \multirow{2}{*}{ Tiempo y ambiente } & bajo & 23 & 44,2 \\
\hline & alto & 29 & 55,7 \\
\hline \multirow{2}{*}{ Regulación del esfuerzo } & bajo & 27 & 51,9 \\
\hline & alto & 25 & 48,0 \\
\hline \multirow{2}{*}{ Aprendizaje paralelo } & bajo & 30 & 57,6 \\
\hline & alto & 22 & 42,3 \\
\hline \multirow{2}{*}{ Búsqueda de ayuda } & bajo & 26 & 50,0 \\
\hline & alto & 26 & 50,0 \\
\hline \multirow{2}{*}{$\begin{array}{l}\text { Estrategias de manejo de } \\
\text { recursos final }\end{array}$} & bajo & 25 & 48,0 \\
\hline & alto & 27 & 51,9 \\
\hline \multirow{2}{*}{ CEAM FINAL } & bajo & 24 & 46,1 \\
\hline & alto & 28 & 53,8 \\
\hline
\end{tabular}


vación como lo mostró el valor final CEAM completo, Fisher $(p<0,001)$; también se encontró asociación con las subescalas el ensayo $(\mathrm{p}=0,001)$ organización $(\mathrm{p}=0,036)$, autorregulación metacognitiva $(\mathrm{p}=0,036)$, aprendizaje paralelo $(\mathrm{p}=0,009)$ y búsqueda de ayu$\mathrm{da}(\mathrm{p}=0,01)$ (Tabla 5).

Se encontró correlación moderada en el uso de estrategias de aprendizaje y motivación con las habilidades investigativas, valor final correlación de Spearman 0,64 (p<0,001) (Tabla 6).

Se encontró asociación entre la motivación, estrategias de aprendizaje y habilidades investigativas de los estudiantes $\mathrm{OR}=0,14$ (IC 95\%: 0,04-0,6) lo que indica que los alumnos que usan estrategias de aprendizaje y motivación tienen 0,14 más probabilidades de tener mejores habilidades investigativas (Tabla 7).

\section{Discusión}

Se validaron dos instrumentos para evaluar habilidades investigativas y estrategias de aprendizaje y motivación con adecuados valores de validez y confiabilidad pudiendo así ser utilizados en poblaciones similares, dentro de las principales limitaciones al validar el instrumento se encontró que no había instrumentos similares que evaluaran las variables en mención, mas si se encontró otros que evaluaban el proceso en diferentes contextos como Ravi y cols.

Tabla 4. Asociación entre el nivel de habilidades investigativas y motivación

\begin{tabular}{|c|c|c|c|c|c|c|}
\hline \multirow{2}{*}{ Motivación* } & & \multicolumn{3}{|c|}{ Habilidades Investigativas } & \multirow{2}{*}{ Total } & \multirow[b]{2}{*}{$\mathrm{p}^{\dagger}$} \\
\hline & & Bajo & medio & alto & & \\
\hline \multirow[t]{2}{*}{ MEC } & bajo & 7 & 23 & 3 & 33 & \\
\hline & alto & 7 & 6 & 6 & 19 & 0,02 \\
\hline Total & & 14 & 29 & 9 & 52 & \\
\hline \multirow[t]{2}{*}{ OEC } & bajo & 9 & 17 & 1 & 27 & \\
\hline & alto & 5 & 12 & 8 & 25 & 0,03 \\
\hline Total & & 14 & 29 & 9 & 52 & \\
\hline \multirow[t]{2}{*}{ VTC } & bajo & 9 & 14 & 1 & 24 & 0,04 \\
\hline & alto & 5 & 15 & 8 & 28 & \\
\hline Total & & 14 & 29 & 9 & 52 & \\
\hline \multirow[t]{2}{*}{ CAC } & bajo & 9 & 16 & 0 & 25 & \\
\hline & alto & 5 & 13 & 9 & 27 & 0,004 \\
\hline Total & & 14 & 29 & 9 & 52 & \\
\hline \multirow[t]{2}{*}{ EPC } & bajo & 10 & 15 & 1 & 26 & \\
\hline & alto & 4 & 14 & 8 & 26 & 0,015 \\
\hline Total & & 14 & 29 & 9 & 52 & \\
\hline \multirow[t]{2}{*}{ MFINLC } & bajo & 9 & 15 & 0 & 24 & \\
\hline & alto & 5 & 14 & 9 & 28 & 0,004 \\
\hline Total & & 14 & 29 & 9 & 52 & \\
\hline
\end{tabular}

${ }^{*} \mathrm{MEC}=$ Motivación intrínseca, OEC= Orientación intrínseca, $\mathrm{VTC}=$ Valor de la tarea, $\mathrm{CAC}=$ Confianza en el aprendizaje, $\mathrm{EPC}=$ Eficiencia Personal MFINLC= Motivación final. $\dagger$ Prueba exacta de Fisher $\mathrm{p}<0,05$
${ }^{10}$ en India donde desarrollaron un instrumento para valorar retos y oportunidades en investigación, Harrison y cols. ${ }^{9}$ en Chile que elaboraron un cuestionario virtual para valorar competencias investigativas en enfermeras y Hollman y cols. ${ }^{9}$ que desarrollaron una encuesta

Tabla 5. Asociación entre el nivel de habilidades investigativas y estrategias de aprendizaje

\begin{tabular}{|c|c|c|c|c|c|c|}
\hline \multirow{2}{*}{$\begin{array}{c}\text { Estrategias } \\
\text { de } \\
\text { aprendizaje* }\end{array}$} & & \multicolumn{3}{|c|}{ Habilidades Investigativas } & \multirow[b]{2}{*}{ Total } & \multirow[b]{2}{*}{$\mathrm{p}^{\dagger}$} \\
\hline & & bajo & medio & alto & & \\
\hline \multirow[t]{2}{*}{ ETC } & bajo & 12 & 12 & 1 & 25 & \\
\hline & alto & 2 & 17 & 8 & 27 & 0,001 \\
\hline Total & & 14 & 29 & 9 & 52 & \\
\hline \multirow{2}{*}{ ELAC } & bajo & 9 & 12 & 1 & 22 & \\
\hline & alto & 5 & 17 & 8 & 30 & 0,045 \\
\hline Total & & 14 & 29 & 9 & 52 & \\
\hline \multirow[t]{2}{*}{ OC } & bajo & 9 & 13 & 1 & 23 & \\
\hline & alto & 5 & 16 & 8 & 29 & 0,036 \\
\hline Total & & 14 & 29 & 9 & 52 & \\
\hline \multirow[t]{2}{*}{ PCC } & bajo & 9 & 12 & 1 & 22 & \\
\hline & alto & 5 & 17 & 8 & 30 & 0,045 \\
\hline Total & & 14 & 29 & 9 & 52 & \\
\hline \multirow[t]{2}{*}{ METAC } & bajo & 9 & 12 & 1 & 22 & \\
\hline & alto & 5 & 17 & 8 & 30 & 0,045 \\
\hline Total & & 14 & 29 & 9 & 52 & \\
\hline \multirow[t]{2}{*}{ EMC TC } & bajo & 9 & 12 & 1 & 22 & \\
\hline & alto & 5 & 17 & 8 & 30 & 0,045 \\
\hline Total & & 14 & 29 & 9 & 52 & \\
\hline \multirow[t]{2}{*}{ TAC } & bajo & 9 & 13 & 1 & 23 & \\
\hline & alto & 5 & 16 & 8 & 29 & 0,036 \\
\hline Total & & 14 & 29 & 9 & 52 & \\
\hline \multirow[t]{2}{*}{ REC } & bajo & 11 & 13 & 3 & 27 & \\
\hline & alto & 3 & 16 & 6 & 25 & 0,052 \\
\hline Total & & 14 & 29 & 9 & 52 & \\
\hline \multirow[t]{2}{*}{ APC } & bajo & 10 & 19 & 1 & 30 & \\
\hline & alto & 4 & 10 & 8 & 22 & 0,009 \\
\hline Total & & 14 & 29 & 9 & 52 & \\
\hline \multirow[t]{2}{*}{ BAC } & bajo & 11 & 14 & 1 & 26 & \\
\hline & alto & 3 & 15 & 8 & 26 & 0,006 \\
\hline Total & & 14 & 29 & 9 & 52 & \\
\hline \multirow[t]{2}{*}{ E.Rec. } & bajo & 11 & 13 & 1 & 25 & \\
\hline & alto & 3 & 16 & 8 & 27 & 0,005 \\
\hline \multirow[t]{2}{*}{ Total } & & 14 & 29 & 9 & 52 & \\
\hline & & & & & & otal \\
\hline \multirow[t]{2}{*}{ CEAM } & bajo & 11 & 13 & 0 & 24 & \\
\hline & alto & 3 & 16 & 9 & 28 & $<0,001$ \\
\hline Total & & 14 & 29 & 9 & 52 & \\
\hline
\end{tabular}

* ETC: Elaboración, ELAC: Ensayo, OC: Organización, PCC: Pensamiento crítico, METAC: Autorregulación Metacognitivas, EMC TC: Metacognitivas, TAC: tiempo y ambiente, REC: Regulación al esfuerzo, APC: Aprendizaje en paralelo, BAC: Búsqueda de ayuda, ERec:Recursos, CEAM: Cuestionario de estrategias de Aprendizaje y Motivación.

$\dagger$ Prueba Exacta de Fisher $\mathrm{p}<0,05$

online que evaluaba: importancia de investigación en odontología, las barreras a la investigación, la participación, y la exposición a la investigación en el plan de estudios dentales.

En el estudio la mayoría de estudiantes refieren haber realizado trabajos de investigación previos lo que coincide con Hollman y cols. ${ }^{11}$ que menciona que el 63\% de los encuestados había realizado investigaciones antes de matricularse y el 54\% de estos estudiantes habían presentado sus proyectos de investigación en un foro fuera de Harvard.

La gran cantidad de respuestas favorables respecto a las habilidades investigativas de los estudiantes señala que poseen un nivel intermedio, lo que coincide con el estudio de Rosales y cols. ${ }^{16}$ realizado en la habana Cuba, siendo mejor valoradas en las etapas de planificación y difusión; las limitaciones se presentan en la etapa de ejecución que al igual que Hollman y cols. ${ }^{11}$ refieren que la enseńanza en bioestadística debería mejorar así como Keib y cols. ${ }^{17}$ que pone énfasis en la aplicación de la práctica basada en la evidencia. En cuanto a publicaciones científicas realizadas el porcentaje es bastante bajo a

Tabla 6. Correlación del Nivel de habilidades investigativas, motivación y estrategias de aprendizaje

\begin{tabular}{ccc}
\hline \multirow{2}{*}{ CEAM } & \multicolumn{2}{c}{ Habilidades Investigativas } \\
\cline { 2 - 3 } & $\mathrm{r}_{\mathrm{s}}{ }^{*}$ & $\mathrm{p}$ valor \\
\hline Motivación Intrínseca & 0,07 & 0,606 \\
Orientación Extrínseca & 0,28 & 0,042 \\
Valor de la Tarea & 0,24 & 0,08 \\
Confianza en el aprendizaje & 0,24 & 0,08 \\
Eficacia personal & 0,45 & 0,001 \\
Escala motivación & 0,44 & 0,001 \\
E. Elaboración & 0,52 & $<0,001$ \\
E. Ensayo & 0,38 & 0,005 \\
E. Organización & 0,3 & 0,031 \\
Pensamiento Crítico & 0,222 & 0,114 \\
E. Metacognitivas & 0,439 & 0,001 \\
E. Manejos de recursos & 0,513 & $<0,001$ \\
E. Tiempo y ambiente & 0,55 & $<0,001$ \\
Regulación del esfuerzo & 0,42 & 0,002 \\
Aprendizaje en paralelo & 0,417 & 0,002 \\
Búsqueda de ayuda & 0,481 & $<0,001$ \\
Escala estrategias de & 0,575 & $<0,001$ \\
aprendizaje & $0,0,001$ \\
Escala CEAM -FINAL & 0,64 &
\end{tabular}

${ }^{*}$ Coeficiente de Spearman

Tabla 7. Determinación del OR del CEAM y Habilidades Investigativas

\begin{tabular}{cccccccc}
\hline & & \multicolumn{2}{c}{ Habilidades Investigativas } & \multirow{2}{*}{ Total } & OR & IC \\
\cline { 2 - 4 } & & bajo & moderado-bueno & & & \\
CEAMFINALC & bajo & 13 & 11 & 24 & \multirow{2}{*}{0,14} & $(0,04-0,60)$ \\
Total & alto & 25 & 3 & 28 & & \\
\hline
\end{tabular}

*CEAM: Cuestionario de Estrategias de aprendizaje y motivación. IC: 95\% 
diferencia de lo reportado por Romesh y cols. ${ }^{7}$ en USA donde 36\% de los estudiantes ya tiene su investigación publicada en revistas indexadas.

Que no haya diferencias significativas en la asociación de habilidades investigativas y aspectos demográficos como aspectos educacionales podría significar que el desarrollo de habilidades investigativas es independiente de la formación de cada persona y pueden haber otros puntos que motiven el desarrollo e interés por la investigación científica como menciona Monirhe y cols. ${ }^{13}$ que los factores ambientales, financiamiento y estructura también son importantes e Imafuku y cols. ${ }^{18}$ que indican que los estudiantes dan mayor atención a los procesos de investigación, incluyendo la autonomía del investigador, la colaboración y los procesos de construcción del conocimiento, sin embargo, coincide con nuestro estudio en el cual hay una tendencia en tener dificultades para llevar a cabo proyectos de investigación en grupo, los cuales el asocia a experiencias de aprendizaje previo, valoración hacia la comunicación interpersonal, la comprensión del proceso de investigación, y las relaciones sociales con los demás.

A diferencia de lo encontrado por Harrison y cols. ${ }^{9}$ donde concluye que si existe diferencia en las habilidades investigativas según el nivel de preparación académica, no se pudo determinar en este estudio puesto que la muestra no fue homogénea.

La mayoría de los estudiantes categorizan la motivación y uso de estrategias de aprendizaje como medio a alto, en la motivación resalta la autoeficacia y motivación extrínseca mientras que en las estrategias de aprendizaje, las estrategias metacognitivas son las más relevantes en coherencia con Monireh y cols. ${ }^{13}$ que resalta el papel mediador de la autoeficacia en investigación en relación a los factores de la investigación y la motivación y como Villegas y cols. ${ }^{12}$ donde la valoración del estudio que hacen los estudiantes, se relacionan directamente con estrategias de organización y regulación metacognitiva, asimismo, se encontró que las principales estrategias que realizan los estudiantes fueron el uso de organizadores e imágenes y subrayado similar a lo que encontró Estrada y cols. ${ }^{19}$ donde los alumnos expresaron que: la lectura repetitiva, resúmenes, esquemas, subrayado, mapas conceptuales son los métodos más utilizados concluyendo que los alumnos de licenciatura cuentan con diversas y variadas técnicas de aprendizaje y a diferencia de Mc. Andrews y cols (EE.
UU.) ${ }^{20}$ que reportó que la lectura repetida fue la estrategia más utilizada con el $83,3 \%$ de los estudiantes.

En nuestro estudio se encontró correlación entre las variables motivación, estrategias de aprendizaje y habilidades investigativas similar a lo reportado por Monireh y cols. ${ }^{13}$ donde los coeficientes de correlación de Pearson mostraron que la mayoría de los factores de autoeficacia, factores de motivación en investigación (motivación interna, motivación externa y sin motivo) y los componentes de factores de investigación (experiencias previas, otros estímulos, experiencias de sustitución, ansiedad y actitud) se correlacionan de manera significativa.

El trabajo evidenció que el uso de estrategias de aprendizaje y motivación en estudiantes de posgrado en odontología tiene 0,14 más probabilidades de desarrollar un mejor nivel de habilidades investigativas lo que coincide con lo desarrollado con Vildoso ${ }^{14}$ en el que refiere que si encontró relación en el uso de estrategias de aprendizaje y autoeficacia personal con las habilidades investigativas de estudiantes de maestría en educación de la UNMSM utilizando cuatro instrumentos de evaluación y como lo referido por Visser y cols. ${ }^{21}$ que dice que los factores motivación, reflexión, participación y la investigación actual, capturan con precisión la percepción de los estudiantes sobre la integración de la investigación.

$\mathrm{Al}$ igual que Knigth y cols. ${ }^{22}$ pensamos que es importante mencionar que el desarrollo de habilidades investigativas es esencial para lograr profesionales de la salud competentes ya que el proceso de investigación se integra con el aprendizaje sobre la salud de la población y los determinantes sociales importantes en salud pública. Así como también la necesidad de incluir la investigación científica con carácter transversal en el currículo de la carrera de odontología como menciona Prieto y cols. ${ }^{23}$.

\section{Conclusiones}

El uso de estrategias de aprendizaje y motivación influye en el nivel de habilidades investigativas de los estudiantes de posgrado en odontología, encontrándose una fuerte asociación y correlación entre las variables. La apreciación global de las habilidades fue de un nivel medio así como el uso de estrategias de aprendizaje y motivación; estos valores se pueden utilizar como una línea basal que permita la evaluación de las mismas variables a futuro. No se encontró di- ferencias significativas entre el nivel de habilidades investigativas y las variables sociodemográficas y educacionales de los estudiantes. Se requieren más estudios con muestras mayores y con aplicación de métodos mixtos y otros diseńos que permitan valorar este proceso dentro de esta misma línea de investigación debido a la complejidad de las variables.

\section{Agradecimientos}

A la UNMSM y a los Doctores: Hans Morgenstern O, Ray Ticse Aguirre, Cesar Aliaga R, Rafael Morales V, Manuel Mattos V, María Pareja V, Raquel Ayón H, Carlos Sotelo E, Raúl Tafur P. por su participación como expertos en la validación de los instrumentos.

\section{Referencias bibliográficas}

1. Accreditation standards for dental education programs. American dental association (2013). Accesible en: http://www.ada.org/ / media/CODA/Files/predoc.ashx. (consultado el 10-01-15)

2. Consejo de evaluación, acreditación, certificación de la calidad de la educación superior Universitaria CONEAU. Estándares para la acreditación de la Carrera profesional universitaria de Odontología.SINEACE; 2010.Disponible en: https://www.sineace.gob.pe/ wp-content/uploads/2015/01/ Estandares_para_la_Acreditacion_ de_la_Carrera_Profesional_Universitaria_de_Odontologia.pdf.

3. Salcedo D, Petkova-Gueorguieva M, Sotomayor J, Delgadillo J, Pineda M, Villavicencio J, Ortiz L, et al. Percepción del clima educacional por estudiantes de odontología en etapa de transición curricular en una muestra peruana. Odontol Sanmarquina. 2014;17(1): 29-34.

4. Emrick J, Gullard A. Integrating research into dental student training: a global necessity. J Dent Res. 2013; 92(12):1053-55.

5. Jaik A, Ortega E. El nivel de dominio de las competencias en metodología de la investigación que poseen los alumnos de posgrado. Tesis doctoral. Instituto Universitario Anglo Español.Cataluña.2012.

6. García MA. La eficiencia terminal en los estudios de posgrado con orientación en investigación UMSNH. Ponencia presentada en el congreso internacional de posgrado en derecho de la UNAM. Recuperado el 5 de abril de 2006. 
Disponible en: http:www.posgrado.unam.mx-derecho.congresoponencias.

7. Romesh PN, Min Kyeong L, Da Silva J, Veerasathpurush A. Impact of a Research Requirement in a Dental School Curriculum. J Den Educ. 2014;78:1364-1371.

8. Grau I, Barciela M, Soto L. Proyección científica de la facultad de estomatología de la universidad de ciencias médicas de la Habana. Rev Cub Estomatol. 2013;50(3):308315.

9. Harrison L, Hernández A, Cianelli R, Rivera M, Urrutia M. Competencias en investigación para diferentes niveles de formación de enfermeras: una perspectiva latinoamericana. Rev Cienc y Enferm. 2005; 11(1): 59-71.

10. Ravi V, Sura S, Sumanthprasad G, Khurana L. Dental students research inventory: A Questionnaire to asses research challenges and opportunities. J Dent Educ. 2010;2(12):1308-18.

11. Holman SD, Wietecha M, Gullard A, Petersen J. Research experiences impact U.S. dental students positive attitudes toward research and science. J Dent Educ. 2014;78(3):334-48.

12. Villegas G, Sotelo L, Sotelo N, Dominguez S. Relación entre estrategias de aprendizaje y motivación en una muestra de estudiantes universitarios de Lima Metropolitana. Rev Psicol. 2011;3(3): 31-44.
13. Monireh S, Hossein K, Mohammad Reza A. The Role of SocialCognitive References in Academic Situations on Students' Research Self-Efficacy and Research Motivation: Testing a Causal Model. Am J Edu Res. 2013;1(3):79-85.

14. Vildoso J. Estrategias de Aprendizaje y Autoeficacia en el Desarrollo de Habilidades investigativas de los Maestristas de la Facultad de Educación de la UNMSM. (tesis para optar el grado de doctora en educación).Universidad Nacional Mayor de San Marcos. Lima-Perú. 2010.

15. Montoya O. Application of the factorial analysis to the investigation of markets. Case of Study. Scientia et Technica Año. 2007;13(35):281-86.

16. Rosales S, Ruano M, Padrón E, Valverde O, Sanz T. Diagnóstico de la formación investigativa en la carrera de estomatología de la facultad Raúl Gonzales Sánchez. Rev Cub Estomatol. 2013;49(2):226238.

17. Keib C, Cailor S, Kiersma M. Chen A. Changes in nursing students' perceptions of research and evidence-based practice after completing a research course. Nurse Educ Tod. 2017;54:37-43.

18. Imafuku R, Saiki T, Kawakami C, Suzuki Y. How do students' perceptions of research and approaches to learning change in undergraduate research?. Int J Med Educ. 2015;12(6):47-55. doi: 10.5116/ijme.5523.2b9e.
19. Estrada-Valenzuela C, PradoMendoza J, De la Fuente-Cabrera L, Navarro-Villalobos M, ElizaldeCorona A, Aguirre-Sarabia N. Eficacia de estrategias de aprendizaje utilizadas por alumnos de la Facultad de Odontología de la Universidad Autónoma de Coahuila, Unidad Torreón. Rev Tamé. 2014;3 (8):264-270.

20. McAndrew M, Morrow CS, Atiyeh L, Pierre GC.J Dental Student Study Strategies: Are SelfTesting and Scheduling Related to Academic Performance? J Dent Educ. 2016;80(5):542-52.

21. Visser-Wijnveen G, van der Rijst $\mathrm{R}$, van Driel J. A questionnaire to capture students' perceptions of research integration in their courses.High Educ. 2016;71(4):473488. doi: 10.1007/s10734-0159918-2.

22. Knight S, Van Wyk J, Mahomed S.Teaching research: a programme to develop research capacity in undergraduate medical students at the University of KwaZulu-Natal, South Africa. BMC Med Educ. 2016;16: 61.

23. Prieto-Benavides D, Palacios A, Cardozo 1, Correa J, Ramírez-Vélez R. Capacidad científica e investigadora de los profesionales de educación en Colombia. Apunts. Edu Fis Dep. 2016; 123(1):19-27. 